\title{
Focusing on fatty acid profile in milk from different species after in vitro digestion
}

\author{
Antonella Santillo*, Lucia Figliola, Maria G Ciliberti, Mariangela Caroprese, Rosaria Marino and \\ Marzia Albenzio \\ Department of the Sciences of Agriculture, Food and Environment (SAFE), University of Foggia, Via Napoli, 25, 71122 Foggia, Italy
}

Received 30 June 2017; accepted for publication 5 April 2018

\begin{abstract}
We report the fatty acid profile of raw milk and of the corresponding digested milk from different sources (human milk, formula milk and donkey, bovine, ovine and caprine milk) to gain information on the nutritional quality of different milk sources in infant nutrition.

Short chain fatty acids (SC-FA) were higher in bovine and caprine milk, intermediate in ovine and donkey and lower in human and formula milk. Medium chain fatty acids (MC-FA) showed the highest values for bovine and caprine milk and the lowest for donkey and formula milk, whereas long chain fatty acids (LC-FA) were the highest in donkey and formula milk and intermediate in human milk.

The percentage distribution of fatty acids liberated after in vitro digestion did not reflect the patterns found in the corresponding milk sources. In particular, MC free fatty acids (MC-FFA) showed the highest and the lowest values in donkey and in formula milk, LC-FFA showed the highest value in human milk. The total FFA was highest in human milk, lowest in formula milk and intermediate in donkey, bovine, ovine, and caprine milk.
\end{abstract}

Keywords: Milk, mammal species, fatty acid, in vitro digestion.

Human milk is considered the best source of nutrients for the infant, being the first food of the life of each newborn mammal and able to fully satisfy energy and nutritional needs for sugars, minerals, vitamins, proteins and lipids. For women who choose not to breastfeed or have insufficient amount of milk, milk-based formula are recommended (Zou et al. 2013). Although production of an identical product to breast milk is not feasible, every effort has been taken to mimic its nutritional profile for normal infant growth and development. Cow milk is most commonly used as the base for such formula milk (Zou et al. 2017). Less usually formula are soy-based or manufactured from other mammalian milks such as buffalo, donkey, sheep, camel, and goat milk (Zou et al. 2013). However, the benefits of using alternative milk sources to human milk still remain unexplored by the dairy industry.

The fat fraction of milk varies among different species especially in terms of structure and size of fat globule, the amount and structure of triacylglycerols (TAG), FFA, phospholipid and cholesterol content (Gantner et al. 2015). Milk fat component has been widely investigated

*For correspondence; e-mail: antonella.santillo@unifg.it in infant nutrition; essential n 3 and n 6 PUFA and their long chain PUFA derivatives such as arachidonic acid and docosahexaenoic acid (DHA) have a central role in cell membrane structure and functions promoting neonatal growth, neurotransmitter metabolism, and visual and nervous system development. Digestion and subsequent bioavailability of lipid and protein components is a complex physico-chemical and enzymatic process; in vitro or ex vivo model digestion is needed to predict the nutritional quality of food products and their constituents (Devle et al. 2014). The molecular form of fatty acids taken up by the enterocyte modulates its incorporation into either the TAG or the phospholipids fraction of chylomicrons as well as its positional distribution in the TAG of chylomicrons (Martin et al. 1993).

In the light of these considerations the aim of the paper was to study the fatty acid profile in milk from different sources (human, formula, donkey, bovine, ovine and caprine) before and after in vitro digestion process, in order to assess if the fatty acid profile of ingested milk reflects the one available after digestion. The investigation of fatty acid profile after in vitro digestion could give information on the availability of these compounds for further intestinal absorption. 


\section{Materials and methods}

\section{Milk sample collection and analysis}

Five lactating women (1-3 months after delivery) were recruited for human milk (HM) collection. Breast milk obtained from complete emptying of the gland in the morning and in the afternoon was pooled from each donor. Liquid commercial formula milk (FM) reported the following composition according to the nutrition label: fat $3 \cdot 6 \%$, total protein $1 \cdot 4 \%$, lactose $5 \cdot 8 \%$. Commercial bovine (BM) and caprine milk (CM) samples were purchased at a local store, while ovine milk (OM) and donkey milk (DM) were taken at a dairy farm located in Foggia (Apulian region, Italy) and pasteurised $\left(63^{\circ} \mathrm{C}\right.$ for $\left.30 \mathrm{~min}\right)$.

Milk samples were analysed for fat, protein, casein, and lactose content using infrared spectrophotometer (MilkoScan FT 120; Foss Electric A/S, Hillerød, Denmark). Each sample was analysed in duplicate.

For total fatty acids analysis in milk and digested milk, lipids were extracted according to O'Fallon et al. (2007). Detailed fatty acids separation method is reported in online Supplementary Materials \& Methods. Fatty acids were grouped into saturated (SFA), monounsaturated (MUFA) and polyunsaturated (PUFA) according to the level of saturation. Atherogenic (Arl) and thrombogenic (TI) indices were calculated according to Ulbricht \& Southgate (1991).

\section{In vitro digestion of milk samples}

All reagents used in the analysis were standard analytical grade. Porcine pepsin (EC $3 \cdot 4 \cdot 23 \cdot 1$ ), porcine pancreatine (EC 3.4.21.4; activity was based on trypsin activity assay) were purchased from Sigma-Aldrich (S.r.I., Milan, Italy) and enzyme activities for the simulation of digestion fluid were determined according to standard protocols. Bile salts concentration was measured using a commercial kit (total bile acid assay kit, STA-631 Cell Biolabs, Inc., San Diego, CA). Simulated salivary fluid (SF) at $\mathrm{pH} 7$, gastric fluid (GF) at $\mathrm{pH} 3$, and intestinal fluid (IF) at $\mathrm{pH} 7$ were prepared with stock solutions of electrolytes, enzymes, $\mathrm{CaCl}_{2}$, and water according to Minekus et al. (2014).

Before in vitro digestion, all milk samples were homogenised and divided into aliquots of $5 \mathrm{ml}$ and analysed in duplicate. The procedure was divided into three steps simulating the oral, gastric and intestinal phases according to Minekus et al. (2014). Modifications of the protocol concerned the oral phase: $\alpha$-amylase was not added to the salivary fluid due to the absence of starch in the analysed samples. Gastric and intestinal phases were conducted in a shaking incubator at $37^{\circ} \mathrm{C}$ for $2 \mathrm{~h}$. Samples were frozen immediately after analysis in liquid nitrogen to slow down enzymatic reactions, and analysed for fatty acids after $24 \mathrm{~h}$.

\section{Free fatty acid analysis in digested milk sample}

Free fatty acids were extracted from digested milk samples according to Devle et al. (2014) and analysed as described in online Supplementary Materials \& Methods.

\section{Statistical analysis}

Total fatty acid composition of milk sources and free fatty acid composition of digested milk sources were processed using ANOVA for repeated measures (SAS Institute, 2011) with milk source as the tested effect. When significant effects were found (at $P<0 \cdot 05$ ), the Tukey's test was used as a post hoc test.

\section{Results}

\section{Chemical composition of milk}

The gross composition of milk from different species and the composition of infant formula are reported in Table 1. Fat content was about $2 \%$ in $\mathrm{HM}$ and below $1 \%$ in DM; regarding ruminant species, fat content was about $4 \%$ in bovine and caprine milk and higher than $8 \%$ in ovine milk. Casein fraction represented almost $80 \%$ of the total protein in ruminant species and about $40 \%$ in $\mathrm{HM}$ and DM. Lactose content showed levels of about $7 \%$ in $\mathrm{HM}$ and DM and values about of $4.5 \%$ in milk from ruminant species.

\section{Fatty acids composition in milk and in digested milk}

The effect of milk source on major groups of fatty acids is reported in Table 2 . In general, differences emerged in the fatty acid profile of milk from different species and of formula milk. Grouping fatty acids according to the length of the carbon chain, it was evident that short chain fatty acids (SC-FA) were higher in bovine and caprine milk, intermediate in ovine and donkey milk and lower in human and

Table 1. Gross composition of milk from different species

\section{Milk source}

\begin{tabular}{|c|c|c|c|c|c|}
\hline Parameter, \% & $\mathrm{HM}$ & DM & $\mathrm{BM}$ & OM & $\mathrm{CM}$ \\
\hline Fat & $2 \cdot 29 \pm 0 \cdot 12$ & $0 \cdot 53 \pm 0 \cdot 01$ & $3 \cdot 63 \pm 0 \cdot 12$ & $8 \cdot 16 \pm 0 \cdot 32$ & $4 \cdot 14 \pm 0 \cdot 15$ \\
\hline Total protein & $1 \cdot 21 \pm 0 \cdot 13$ & $1 \cdot 46 \pm 0.04$ & $3 \cdot 53 \pm 0 \cdot 56$ & $6 \cdot 01 \pm 0 \cdot 48$ & $3 \cdot 22 \pm 0 \cdot 35$ \\
\hline Casein & $0 \cdot 48 \pm 0 \cdot 21$ & $0 \cdot 57 \pm 0 \cdot 02$ & $2 \cdot 72 \pm 0 \cdot 10$ & $4 \cdot 56 \pm 0 \cdot 13$ & $2 \cdot 29 \pm 0 \cdot 12$ \\
\hline Lactose & $7 \cdot 49 \pm 0 \cdot 13$ & $6 \cdot 78 \pm 0 \cdot 15$ & $4 \cdot 69 \pm 0 \cdot 10$ & $4 \cdot 37 \pm 0 \cdot 11$ & $4 \cdot 18 \pm 0 \cdot 12$ \\
\hline
\end{tabular}

n.d., not detectable; HM, human milk; DM, donkey milk; BM, bovine milk; OM, ovine milk; CM, caprine milk 
Table 2. Effect of milk source on fatty acid composition in raw milk and on free fatty acids composition of digested milk

Milk source

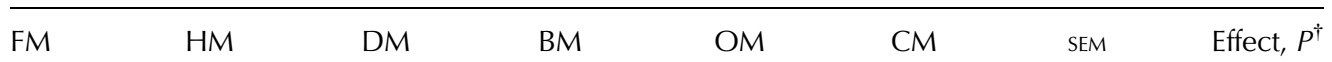

Milk

Fatty acids, \% FAME
SC-FA
MC-FA
LC-FA
S-FA
MU-FA
PU-FA

Digested milk

Fatty acids, $\mu \mathrm{g} / \mathrm{ml}$ of extract

$\begin{array}{lrrr}\text { SC-FFA } & 31 \cdot 90 \mathrm{a} & 36 \cdot 33 \mathrm{a} & 14 \cdot 28 \mathrm{~b} \\ \text { MC-FFA } & 78 \cdot 40 \mathrm{c} & 125 \cdot 88 \mathrm{~b} & 136 \cdot 68 \mathrm{a} \\ \text { LC-FFA } & 84 \cdot 23 \mathrm{~b} & 120 \cdot 47 \mathrm{a} & 98 \cdot 18 \mathrm{~b} \\ \text { S-FFA } & 163 \cdot 83 & 231 \cdot 67 & 240 \cdot 50 \\ \text { MU-FFA } & 12 \cdot 32 \mathrm{~b} & 35 \cdot 00 \mathrm{a} & 2 \cdot 60 \mathrm{c} \\ \text { PU-FFA } & 18 \cdot 39 & 16 \cdot 00 & 6 \cdot 03 \\ \text { Total FFA } & 194 \cdot 53 \mathrm{c} & 282 \cdot 68 \mathrm{a} & 249 \cdot 14 \mathrm{~b}\end{array}$

$\begin{array}{rrr}21 \cdot 20 b & 17 \cdot 54 c & 26 \cdot 20 a \\ 48 \cdot 15 a & 39 \cdot 48 b & 44 \cdot 95 a \\ 30 \cdot 65 d & 42 \cdot 98 c & 28 \cdot 85 d \\ 73 \cdot 90 a & 65 \cdot 15 b & 77 \cdot 26 a \\ 22 \cdot 25 e & 27 \cdot 21 d & 19 \cdot 15 e \\ 3.91 d & 7 \cdot 69 d & 3 \cdot 64 d\end{array}$

$\begin{array}{ll}0 \cdot 32 & * * * \\ 0 \cdot 51 & * * * \\ 0 \cdot 74 & * * * \\ 0 \cdot 73 & * * * \\ 0 \cdot 70 & * * * \\ 0 \cdot 64 & * * *\end{array}$

$32 \cdot 74 a$
$127 \cdot 46 b$
$84 \cdot 35 b$
$229 \cdot 88$
$4 \cdot 64 c$
$9 \cdot 98$
$244 \cdot 54 b$

$43 \cdot 42 a$
$108 \cdot 82 b$
$97 \cdot 84 b$
$218 \cdot 79$
$11 \cdot 73 b$
$19 \cdot 56$
$250 \cdot 08 b$

$24 \cdot 45 b$
$121 \cdot 04 b$
$91 \cdot 04 b$
$202 \cdot 90$
$10 \cdot 43 b$
$22 \cdot 92$
$236 \cdot 52 b$

$\begin{array}{rl}4 \cdot 67 & * \\ 3 \cdot 75 & * * * \\ 6 \cdot 28 & * \\ 10 \cdot 48 & \text { NS } \\ 2 \cdot 51 & * * * \\ 5 \cdot 75 & \text { NS } \\ 13 \cdot 97 & * *\end{array}$

FM, formula milk; HM, human milk; DM, donkey milk; BM, bovine milk; OM, ovine milk; CM, caprine milk SC-FA = short chain fatty acids; SC-FFA = short chain free fatty acids; $M C-F A=$ medium chain fatty acids; MC-FFA = medium chain free fatty acids; LC-FA = long chain fatty acids; LC-FFA = long chain free fatty acids; S-FA = saturated fatty acids; S-FFA = saturated free fatty acids; MU-FA = monounsaturated fatty acids; MU-FFA = monounsaturated free fatty acids; PU-FA = polyunsaturated fatty acids; PU-FFA = polyunsaturated free fatty acids; Total FFA = total free fatty acids.

$\dagger$ NS, $P<0.05 ; * P<0 \cdot 05 ;{ }^{* *} P<0 \cdot 01 ; * * * P<0 \cdot 001$. a, b,c,d: Within a row, values with different suffixes differ significantly, $\mathrm{P}<0.05$ or greater

formula milk. Medium chain fatty acids (MC-FA) showed the highest values for bovine and caprine milk, intermediate in human and ovine milk and the lowest for donkey and formula milk. Long chain fatty acids (LC-FA) were the highest in donkey and formula milk. Among SC-FA, capric acid was the most abundant in milk from ruminant species, particularly caprine, and from donkey milk whereas this fatty acid was about 5 and 13 time lower in human milk and infant formula than in bovine milk. Lauric acid was the most represented among the SC-FA in formula and in human milk (detailed FA composition of milk is reported in online Supplementary File Table S1). Myristic acid and palmitic acid were the most represented in MC-FA and their value was about 3 and 2.5 times lower in infant formula and donkey milk compared to bovine milk, respectively. In general regarding LC-FA the most representatives were C18:0, C18:1 cis-9 and C18:2. In particular, donkey milk was also characterised for a high content of C18:3n 3 and C24:1. Furthermore human milk showed eicosapentaenoic acid (EPA) and DHA values greater than milk from other species and formula milk.

Higher values of S-FA were found in caprine and bovine and lower for formula milk, maternal and donkey milk, intermediate for ovine milk. Infant formula showed the highest MU-FA, intermediate in human and donkey milk whereas lower values in milk from ruminant species. Lowest PU-FA was found in milk from ruminants, intermediate in formula and human milk and the highest in donkey milk.

Arl and TI were higher in milk from ruminant species than in the other milk sources. In particular, caprine milk showed the highest values whereas ovine milk was under the value of 3 for both indexes.

In order to evaluate the impact of in vitro digestion on the liberation of free fatty acids from different milk sources, comparison of percentage distribution of free fatty acids in digested milk and total fatty acids distribution of the corresponding milk sources was done and the rate of liberation of fatty acids was calculated as the per cent ratio of free fatty acids and total fatty acids content. The percentage distribution of total S-FA, MU-FA and PU-FA in milk and the percentage distribution of free S-FA, MU-FA and PU-FA in digested milk from different sources are reported in Fig. 1. FM, HM, and DM showed a major liberation of S-FA vs. MU-FA and PU-FA. On the contrary milk from ruminant species evidenced a major contribute of S-FA and the liberation of MU-FA after digestion turned out to be limited with an average percentage of $3 \%$.

The effect of milk source on major groups of FFA of digested milk is reported in Table 2. The FFA grouped according to the length of the carbon chain highlighted significant differences in short-, medium-, and long-chain FFA. Regarding the SC-FFA, donkey and caprine milk showed the lowest content and C6:0 was not detected at all (detailed FFA composition of digested milk is reported in online Supplementary File Table S2). Furthermore, the highest level of C12:0 was found in human milk upon digestion whereas intermediate level was ascribed to formula milk.

Medium-chain FFA showed the highest and the lowest values in donkey and in formula milk, respectively; accordingly C16:0 showed the same behaviour, representing the 


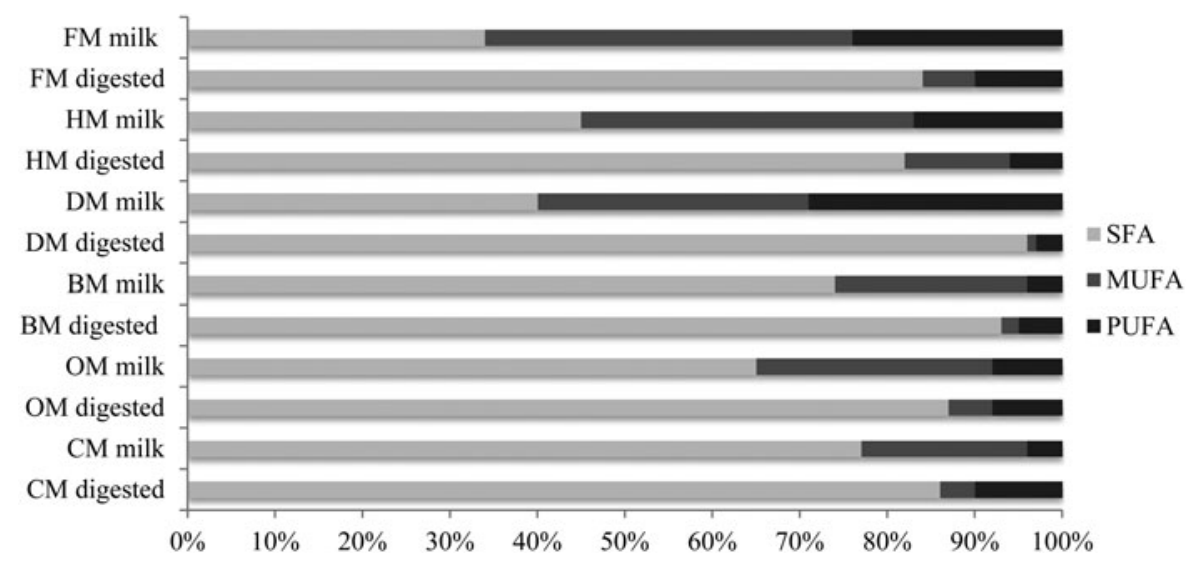

Fig. 1. Percentage distribution of total S-FA, MU-FA and PU-FA in milk and percentage distribution of free S-FA, MU-FA and PU-FA in digested milk from different sources. FM, formula milk; HM, human milk; DM, donkey milk; BM, bovine milk; OM, ovine milk; CM, caprine milk; saturated fatty acids (S-FA); monounsaturated fatty acids (MU-FA); polyunsaturated fatty acids (PU-FA).

most abundant among MC-FFA. Furthermore, free C14:0 had the highest values in human and caprine milk, whereas C16:1 was detected only in human, bovine, and caprine milk with the highest level found in the former milk. The total LC-FFA showed the highest value in human milk which also showed the highest content for C18:1 cis-9, C18:2 cis-9, cis-12, C22:0, C24:1. Apart from the milk source analysed, C18:0 represented more than $50 \%$ of the whole class of LC-FFA. In particular, in donkey milk almost the total LC-FFA was represented by stearic acid. Monounsaturated FFA were significantly different in the digested milks, with the highest value found in human milk with the major contribute of oleic acid. This FFA was six times higher in human than in digested milk from animal species, and about three fold higher than in formula milk. Furthermore, it is worth noting that, although the difference was not significant, EPA and DHA were always found in human digested milk and were absent from bovine and donkey milk. Finally the total FFA were highest in human milk, lowest in formula milk and intermediate in donkey, bovine, ovine, and caprine milk.

\section{Discussion}

\section{Chemical composition of milk}

In general, data for chemical composition of milk from different species were in accordance with reference literature (Gantner et al. 2015; Claeys et al. 2014); however, fat content of human milk from the present study showed lower values than previously reported for the same milk. It is known that in human milk fat varies much more than any other major constituents due to stage of lactation, prematurity, changes during a feed and during the day, age and parity and especially due to maternal diet and weight gain during pregnancy. In general human and donkey milk showed a closer similarity, whereas milk from ruminant species showed mean values of fat and protein double those found in human and donkey milk; in particular, ovine milk was confirmed to be the richest in both nutritional components. The lower casein content in human milk is in accordance with its peculiar protein distribution. Non-protein nitrogen is a remarkable fraction in human milk compared to other milk source, representing over 20-25\% (Armaforte et al. 2010) and consists mainly of free amino acids and urea, peptides and ammonium, polyamines and nucleotides (Claeys et al. 2014). Finally lactose is the most abundant carbohydrate, whatever milk source, providing energy, and usually it is added into formula to standardise the lactose concentration into the human milk range (Zou et al. 2017).

\section{Fatty acids composition in milk}

The fatty acid pattern in human milk derives from endogenous synthesis in the mammary gland and uptake from maternal plasma and both sources are influenced by maternal nutrition (Innis, 2014). Fatty acid composition of milk fat in non-ruminant species tends to only reflect the lipid composition of diet, whereas diet has a minor impact on fatty acid profile in milk from ruminant species due to the ruminal biohydrogenation processes that modify the fatty acid profile of base food. It is worth noting that among ruminant species, PU-FA in ovine milk showed a mean value double that in caprine and bovine milk likely due to the feeding system being based on pasture and fresh forage (Nudda et al. 2014) and to the specific activity of $\Delta^{9}$ desaturase.

Human milk contains naturally long chain PU-FA n 3 and n 6 series and formula milk is generally enriched with essential nutrients such as EPA and DHA, with beneficial effects on proper brain and visual development in the fetus and maintenance of neural and retinal photoreceptors throughout life (Zou et al. 2017). Apart from the milk source analysed, the most represented LC-FA C18:1 cis-9 and C18:2 are of interest in human nutrition. The former is an important source of energy for the baby, and the latter an essential fatty acid 
precursor of other long chain fatty acids (Lopez-Huertas, 2010). As a consequence of fatty acid profile atherogenic and thrombogenic indexes were more favourable to human nutrition in formula, human and donkey milk.

\section{Fatty acids composition in digested milk}

Lipid digestion is characterised by different steps which occur firstly in the stomach and secondly in the small intestine where the bile salts emulsify intermediate products, making them available to pancreatic lipases that hydrolyse the bonds in the sn-1 and sn-3 position by releasing a 2monoacylglycerol (Gallier et al. 2013a; Devle et al. 2014). In all the milk sources subjected to the study, the percentage distribution of fatty acids liberated upon gastrointestinal digestion did not reflect the patterns found in the corresponding milk sources. In particular the most important changes were observed in $\mathrm{FM}, \mathrm{HM}$, and DM with a major liberation of S-FA, whereas comparable fatty acids profiles were observed in milk and in the corresponding digested milk from ruminant species, where the predominant group is always represented by S-FA.

Differences in the FFA of digested milk sources could be attributed to multiple factors concerning the interaction between milk substrate and digestion process, such as the presence of endogenous enzymes to milk, the different structure and organisation of the milk fat globules, and the position of bounds in TAG. However, similarities between acidic profile of human milk and milk from other animal sources after digestion are relevant for the industrial exploitation of the latter milk sources for their use as basic components in infant formula more respondent to human milk profile.

Milk, and particularly human milk, contains lipolytic enzymes including lypoproteinlipase (LPL) and bile saltsimulated lipase (BSSL). LPL is an enzyme similar to pancreatic lipase which is stimulated by bile salts (de Oliveira et al. 2016). Both LPL and BSSL are considered to be crucial for the digestion of lipids by human babies, as they secrete low levels of both pancreatic lipase and bile salts (Fox \& Kelly, 2006). In contrast to human milk, infant formula is devoid of any BSSL activity and a consequent reduction in hydrolysis ester bonds of TAG may be expected (Martin et al. 1993). The size and structure of the fat globules affect digestion; the smaller the fat globules the more efficient is the process (Claeys et al. 2014). On the other hand, the size and dispersion of the fat globules confer greater consistency to goat and sheep milk favoring freezing without phase separation (Balthazar et al. 2017), with positive implications on their industrial exploitation. In particular, goat milk has smaller globules (diameter of about $3 \mu \mathrm{m}$ ) than sheep (about $3.5 \mu \mathrm{m}$ ) and bovine (about $4.8 \mu \mathrm{m}$ ) (Gantner et al. 2015); human milk has similar dimensions to donkey milk (about $4 \mu \mathrm{m}$ ) while infant formula is characterised by a very small dimension of milk fat globules (about $0 \cdot 4 \mu \mathrm{m}$ ) (Nguyen et al. 2015). The structural characteristics of milk fat globules membrane (MFGM) is another notable factor that influences the different digestibility of fat. The MFGM in human and donkey milk consists of three layers, the internal protein layer, intermediate phospholipids layer and the external layer of glycoproteins on which are bound branched oligosaccharides (Ye et al. 2011). In bovine milk the external layer is further coated with phospholipids, and pancreatic lipase has more difficulty reaching the triglyceride core (Gallier et al. 2013a, b). Recent reports highlight the negative consequences on infant's health of formula supplemented with vegetable oil as a major source of fat, since these formulas specifically lack MFGM (Zou et al. 2017).

The S-FA in human milk are located mainly in sn-2 position which is unfavourable for the action of lipolytic enzymes. Accordingly, the rate of liberation of C12:0, C14:0, C16:0, and C18:0 was lower than that reported for the corresponding fatty acids in milk from ruminant species. However, the highest level of $\mathrm{C} 12: 0$ in human milk upon gastro-intestinal digestion and the intermediate level in formula milk were in accordance with the higher content of lauric acid in the corresponding milk sources. The amount of free S-FA in digested human milk may be a balance between the preferential position occupied by those fatty acids and the size of the fat globules. It is reported that in human milk C16:0 is located mainly in the sn-2 position whereas in bovine, ovine, and caprine milk it is primarily found in sn-1 position (Claeys et al. 2014); however the 2-monoglycerides with palmitic acid at the sn-2 position are easier to absorb by infant than free fatty acids (Sidnell \& Greenstreet, 2011). The highest level C16:1 found in human milk may be ascribed to the sn- 1 and sn-3 position in TAG. Overall, the physiological significance of MC-FA relys on their rapid hydrolysis and absorption by the epithelial barrier and their transport through the bloodstream where they are rapidly catabolised (Mills et al. 2011). The higher level of LC-FFA found in human milk maybe ascribed to the binding site at sn-3 position whereas it is reported that mainly SC-FA and MC-FA are bound at the sn-3 position in bovine milk (Nguyen et al. 2015).

\section{Conclusion}

The analysis of the fatty acid profile in human milk, formula milk and different animal species showed that SC-FA were higher in bovine and caprine milk, intermediate in ovine and donkey milk, and lower in human and formula milk. The group of MC-FA showed the highest values for bovine and caprine milk and the lowest for donkey milk and formula milk, whereas LC-FA were the highest in donkey and formula milk and intermediate in human milk. Infant formula and human milk showed higher percentage of MU-FA and PU-FA than other milk sources.

The percentage distribution of fatty acids liberated upon gastrointestinal digestion did not reflect the patterns found in the corresponding milk sources likely due to the different susceptibility of milk sources to the action of the 
gastrointestinal enzymes used in the in vitro digestion process. The amount of total FFA was highest in human milk, lowest in formula milk and intermediate in donkey, bovine, ovine, and caprine milk. In particular, the total LC-FFA and MU-FFA were highest in human milk due to the major contribute of oleic acid.

The study of fatty acid pattern liberated upon milk digestion is useful in the exploitation of alternative milk sources for design and optimisation of innovative substitutes in infant feeding and may be further associated to the study of fatty acids bioavailability upon intestinal absorption.

\section{Supplementary material}

The supplementary material for this article can be found at https://doi.org/10.1017/S0022029918000274

\section{References}

Armaforte E, Curran E, Huppertz T, Ryan CA, Caboni MF, O'Connor PM, Ross RP, Hirtz C, Sommerer N, Chevalier F \& Kelly AL 2010 Proteins and proteolysis in pre-term and term human milk and possible implications for infant formulae. International Dairy Journal 20 715-723

Balthazar CF, Pimentel TC, Ferrão LL, Almada CN, Santillo A, Albenzio M, Mollakhalili N, Mortazavian AM, Nascimento JS, Silva MC, Freitas MQ, Sant'Ana AS, Granato D, \& Cruz AG 2017 Sheep milk: physicochemical characteristics and relevance for functional food development. Comprehensive Reviews in Food Science and Food Safety 16 247-262

Claeys WL, Verraes C, Cardoen S, De Block J, Huyghebaert A, Reas K, Dewettinck K \& Herman L 2014 Consumption of raw or heated milk from different species: an evaluation of the nutritional and potential health benefit: a review. Food Control 42 188-201

De Oliveira SC, Bourlieu C, Ménard O, Bellanger A, Henry G, Rousseau F, Dirson E, Carrière F, Dupont D \& Deglaire A 2016 Impact of pasteurization of human milk on preterm newborn in vitro digestion: gastrointestinal disintegration, lipolysis and proteolysis. Food Chemistry 211 171-179

Devle H, Ulleberg EK, Naess-Andresen CF, Rukke EO, Vegarud G \& Ekeberg D 2014 Reciprocal interacting effects of proteins and lipids during ex vivo digestion of bovine milk. International Dairy Journal 36 $6-13$

Fox PF \& Kelly AL 2006 Indigenous enzymes in milk: overview and hystorical aspects - part 1. International Dairy Journal 16 500-516

Gallier S, Zhu XQ, Rutherfurd SM, Ye A, Moughan PJ \& Singh H 2013a In vivo digestion of bovine milk fat globules: effect of processing and interfacial structural changes. II. Upper digestive tract digestion. Food Chemistry 141 3215-3223
Gallier S, Cui J, Olson TD, Rutherfurd SM, Ye A, Moughan PJ \& Singh H $2013 \mathrm{~b}$ In vivo digestion of bovine milk fat globules: effect of processing and interfacial structural changes. I. Gastric digestion. Food Chemistry 141 3273-3281

Gantner V, Mijic P, Baban M, Skrtic Z \& Turalija A 2015 The overall and fat composition of milk of various species: a review. Mljekarstvo 65223 231

Innis SM 2014 Impact of maternal diet on human milk composition and neurological development of infants. The American Journal of Clinical Nutrition 99 734-741

Lopez-Huertas E 2010 Health effects of oleic acid and long chain omega-3 fatty acids (EPA and DHA) enriched milks. A review of intervention studies. Pharmacological Research 61 200-207

Martin JC, Bougnoux P, Antoine JM, Lanson M \& Cuet C 1993 Triacylglycerol structure of human colostrum and mature milk. Lipids 28 637-643

Mills S, Ross RP, Hill C, Fitzgerald GF \& Stanton C 2011 Milk intelligence: mining milk for bioactive substances associated with human health. International Dairy Journal 21 377-401

Minekus M, Alminger M, Alvito $P$, Ballance $S$, Bohn T, Bourlieu C, Carrière F, Boutrou R, Corredig M, Dupont D, Dufour C, Egger L, Golding M, Karakaya S, Kirkhus B, Le Feunteun S, Lesmes U, Macierzanka A, Mackie A, Marze S, McClements DJ, Ménard O, Recio I, Santos CN, Singh RP, Vegarud GE, Wickham MS, Weitschies W \& Brodkorb A 2014 A standardized static in vitro digestion method suitable for foodAn international consensus. Food \& Function 5 1113-1124

Nguyen TTP, Bhandari B, Cichero J \& Prakash S 2015 A comprehensive review on in vitro digestion of infant formula. Food Research International Journal 76 373-386

Nudda A, Battacone G, Bonaventura ON, Cannas A, Francesconi AHD Atzori AS \& Pulina G 2014 Feeding strategies to design the fatty acid profile of sheep milk and cheese. Revista Brasileira de Zootecnia 43 445-456

O'Fallon JV, Busboom JR, Nelson ML \& Gaskins CT 2007 A direct method for fatty acid methyl ester synthesis: application to wet meat tissues, oils, and feedstuffs. Journal of Animal Science 85 1511-1521

SAS Institute 2011 SAS User's Guide: Statistics. Version 9.2 ed. Cary, NC: SAS Inst. Inc.

Sidnell A \& Greenstreet E 2011 Infant nutrition-review of lipid innovation in infant formula. Nutrition Bulletin 36 373-380

Ulbricht TLV \& Southgate DAT 1991 Coronary heart disease: seven dietary factors. Lancet 338 985-992

Ye A, Cui J \& Singh H 2011 Proteolysis of milk fat globule membrane proteins during in vitro gastric digestion of milk. Journal of Dairy Science $942762-2770$

Zou X, Huang J, Jin Q, Guo Z, Lin Y, Cheong L, Xu X \& Wang X 2013 Lipid composition analysis of milk fats from different mammalian species: potential for use as human milk fat substitutes. Journal of Agricultural Food Chemistry 61 7070-7080

Zou X, Ali AH, Abed SM \& Guo Z 2017 Current knowledge of lipids in human milk and recent innovations in infant formulas. Current Opinion in Food Science 16 28-39 